\title{
Title: A Systematic Review of the Evidence on Inflammation in Depressive Illness and Symptoms in in Chronic and End Stage Kidney Disease
}

Simone Jayakumar ${ }^{1}$, Stacey Jennings ${ }^{1}$, Kristoffer Halvorsrud ${ }^{2}$, Cristopher Clesse ${ }^{1}$, Livia A. Carvalho $^{3}$, Kamaldeep Bhui. ${ }^{1,4}$

${ }^{1}$ Center for Psychiatry, Wolfson Institute of Preventive Medicine, Barts \& The London School of Medicine \& Dentistry. Queen Mary, University of London, Old Anatomy Building, Charterhouse Square, London EC1M 6BQ.

${ }^{2}$ North Thames ARC, University College London

${ }^{3}$ Department of Clinical Pharmacology, William Harvey Research Institute. Barts \& The London School of Medicine \& Dentistry. Queen Mary, University of London.

${ }^{4}$ Department of Psychiatry and Nuffield Department of Primary Care Health Sciences, University of Oxford. Hon. Consultant Psychiatrist, East London NHS Foundation Trust. 


\begin{abstract}
Background

Depressive illness and symptoms are known to be common in physical health problems and are present in at least a third of people with chronic kidney disease and end stage renal failure (CKD/ESKD). Depressive illness and symptoms in CKD/ESKD complicates care, is associated with a shorter life expectancy, and may arise in response to inflammation.

\section{Methods}

We undertook a systematic review of studies including measures of depression and inflammatory markers in CKD/ESKD. The protocol was pre-registered with PROSPERO: CRD42019141305. Data base searches were completed in 2019 and repeated in May 2020. We assessed study quality, and undertook a narrative synthesis as well as meta-analyses of cross-sectional and longitudinal studies, examining associations between depressive states and inflammatory markers.
\end{abstract}

\title{
Results
}

There was significant heterogeneity in study quality, comorbidities, samples, depression measures, and study design, as well as in the specific measured inflammatory markers. Overall, there is some evidence for associations of IL6 and CRP with depressive illness and symptoms, with inconclusive or contradictory evidence for other inflammatory markers. There were few intervention studies. Studies of samples with physical comorbidity and higher rated quality were more likely to show positive associations. There were few longitudinal studies.

\section{Conclusions}

There is some evidence of inflammation being an important correlate of depressive illness and symptoms in the presence of physical health comorbidities. Better research designs are needed including a range of inflammatory markers. 


\section{Introduction}

Chronic Kidney Disease (CKD) is a decline of the kidney function for over three months , and affects $9.1 \%$ of the world population. It's most severe stage is named End Stage Kidney Disease (ESKD), with patients needing hemodialysis, peritoneal dialysis or awaiting kidney transplant, affects $0.041 \%$ of the population ${ }^{2,3}$. The prevalence of CKD and ESKD has been rising over the last 18 years and is anticipated to double by $2030-2040^{45}$. The increase in prevalence is explained mainly by increasing rates of etiological comorbidities, such as diabetes (type 1 and 2) and hypertension ${ }^{3}$ and, to a lesser extent HIV, other infectious diseases, and external conditions such as agricultural occupation and heavy metal exposure ${ }^{67}$.

Estimates suggest $21.4 \%-26.5 \%$ of CKD patients and $22.8 \%-39 \%$ of ESKD patients suffer depressive symptoms 8 , and consequently face poorer clinical outcomes and higher mortality rates 910 . Diagnosing and treating depression in this population is not trivial. There may be a difference in the diagnosis, epidemiology, and treatment of depression between CKD and ESKD. It is possible that risk factors, epidemiology, and treatment of depression in CKD is more similar to the general population; whereas in ESKD guidance is inconclusive due to small sample sizes, different etiology of disease, lack of control group, selection and drop-out biases. The evidence for use of antidepressant medication in patients with kidney disease is poor. A recent global systematic review found only four randomized controlled trials of SSRIs yielding inconsistent results. A recent study in the UK also found sub-optimal practice patterns relating to the management of antidepressant medication in the dialysis population. It begs the need for both better evidence and innovative approaches.

There is now growing evidence that inflammation is likely to be causally linked to the development of depression. If proven, inflammation could be a target for prevention and treatment of depression. While comorbidity of CKD and ESKD with depression is evident, it is 
unclear whether inflammation is a shared underlying mechanism, to what extent inflammation differs from people with depression but without chronic physical illness and which type of inflammatory markers are observed. We have therefore conducted a systematic review of the literature on inflammation and depression in patients with CKD and ESKD.

\section{Methods}

The review was conducted in accord with Preferred Reporting Items for Systematic Reviews and Meta-Analyses (PRISMA) guidelines ${ }^{15}$, and registered on PROSPERO on $28^{\text {th }}$ August $2019\left(\right.$ CRD42019141305) ${ }^{16}$

Inclusion and exclusion criteria

Inclusion and exclusion criteria are set out using the Population, Exposure, Comparison, Outcome (PECO) framework ${ }^{17}$. The criteria were developed with clinical and research experts and PPI representatives. The review includes studies of (a) adult patients ( $\geq 18$ years) with ESRD or CKD, (b) inflammatory biomarkers known to be associated with depression (c) depression measured by standardised clinical interviews, or by administered or self-report psychometric instruments. We included case control, cohort (prospective and retrospective), and cross-sectional studies; and non-randomised and randomised controlled trials (RCTs). Exclusion criteria included animal studies, non-English language studies, studies published in non-peer reviewed journals; given the sparsity of evidence, we excluded studies of kidney transplant or acute kidney injury patients. 
Search strategy

A sample search is shown in supplement document 1. The search was run in May 2019 and rerun in September 2020. No new studies were identified. We searched electronic databases (PubMed, Embase, MEDLINE, PsychINFO/PsychArticles, Scopus, Web of Science, CINAHL and Cochrane CENTRAL) for published studies up to May 2019, using a search strategy developed with an information scientist. To optimise the capture of relevant research, the first 200 relevant publications from Google Scholar were also considered ${ }^{18}$. Controlled (MESH terms) and text entries were used across all electronic databases. Databases such as DARE, ETHOS, OATD, NICE and PROSPERO were checked for existing or ongoing reviews prior to database searches. Forward and backward citation tracking was undertaken for studies that entered the review and progressed to data extraction.

Study selection

References, titles and abstracts from electronic database searches were exported into Rayyan QCRI ${ }^{19}$. Titles and abstracts were screened independently by two reviewers (SJ and JS). Screening of titles and abstracts against the inclusion and exclusion criteria was piloted on the first 100 abstracts and revealed that in $50 \%$ of publications, information on inflammatory biomarkers was not mentioned in the abstract. Therefore, abstracts meeting all other inclusion criteria underwent full text screening. Full text articles were checked independently by SJ and JS against the inclusion and exclusion criteria. Authors of studies and potentially relevant conference abstracts were contacted to secure full text articles. After one month to respond, one recontact was attempted. Inter-rater reliability for the full-text screening indicated good agreement between reviewers ( $\kappa$ 0.89). Uncertainties were resolved by consensus and unresolved discrepancies were referred to a third reviewer (KB). 


\section{Data extraction}

A data extraction table was piloted on 10 studies and refined (supplement document 2). Study data were extracted independently by two reviewers (SJa and SJe) and charted in an Excel spreadsheet. Study characteristics extracted included study authors, year published, sample description, methodological characteristics, demographics (age, gender, ethnicity), depression definition and cut-off scores, type and levels of inflammatory biomarkers and main results. Statistical information extracted included means/medians, standard deviations/range/interquartile range for outcome and exposure of interest, $\mathrm{N}$ for depressed and non-depressed groups, test statics and p-values. Discrepancies were discussed and resolved between the two reviewers $(95.2 \%$ agreement). If data was not available on the outcome/exposure of interest, then corresponding authors were contacted and those that responded within a month were included in the meta-analysis.

Methodological quality and risk of bias

The risk of bias was assessed independently by two reviewers (SJe and SJa). RCT's were assessed using the 'Risk of Bias $2^{`}(\operatorname{RoB} 2)^{20}$, which assesses risk of bias for a single outcome across five domains (randomisation, deviations from intended interventions, missing outcome data, measurement of outcome, and selection of reported result). It also provides an overall judgement about risk of bias for each domain as well as overall risk of bias judgement. Nonrandomised interventional studies (NRIS) were assessed using the 'Risk Of Bias In Nonrandomized Studies - of Interventions' (ROBINS-I) ${ }^{21}$. ROBINS-I contains seven domains through which risk may be introduced into a study. Responses to each of the items provide 
domain-level judgements about bias; using these we made an overall judgement of bias for a particular outcome (low risk, moderate risk, serious risk and critical risk of bias).

The Newcastle-Ottawa Scale (NOS) was used to assess the quality of cohort studies (CS) ${ }^{22}$. This assigns up to nine stars across three domains: 1) selection of study groups (4 stars); 2) comparability of groups (2 stars), and 3) ascertainment of exposure and outcomes (3 stars). Visually, more stars reflect less bias and study quality is rated as good, fair, or poor. In addition, studies were awarded one point for ascertainment of exposure if information was provided on how inflammatory biomarkers were measured (i.e. blood samples or medical records). For assessment of outcome, studies were awarded one star if depression was diagnosed through a structured clinical interview or if data was obtained from medical records; no stars were allocated for self-report measures or no description.

Cross-sectional studies (CSSs) were assessed using the AXIS tool designed specifically for $\mathrm{CSSs}^{23}$. The tool includes twenty questions relating to study design, sample size justification, target population, sampling frame, sample selection, measurement validity and reliability, and overall methods as opposed to the interpretation (e.g. discussion and conclusion) of the study. The tool does not provide a numerical score but allows the reviewer to assess each individual aspect of study design based on to give an overall judgment of study quality. Cohort studies from which cross-sectional data from one time point were used were assessed on the AXIS tool.

\section{Data analysis}

Narrative synthesis

Sixty-one RCT and non-RCT publications were included in the narrative synthesis, conducted in accord with established guidance ${ }^{24}$. Initially the preliminary synthesis was developed by 
tabulation of study design, aims, study location, total sample, mean age $\pm \mathrm{SD}, \mathrm{n}$ and $\%$ women, depression definition, $\mathrm{n}$ and $\%$ of patients with depression, specific inflammatory biomarkers, summary of main findings, as well as information about interventions and follow up time points (if relevant). The main overarching thematic conclusions about depression and inflammation were identified across the studies and subcategorized according to specific inflammatory biomarkers. Inflammatory markers reported within the theme and whether the study reported a significant or non-significant result for the specified outcome. Studies reporting data on IL-1 inflammatory marker were not included as none specified the type of IL-1 marker.

Following familiarisation and comparison for similarities and contrasts, preliminary synthesis was performed to capture relationships between individual characteristics of studies and the findings. This was done by looking at heterogeneity in the included studies, which involved detailed inspection of sample sizes, sample characteristics, study location, depression definition as well as patient inclusion and exclusion criteria. Finally, the strength of evidence provided by the studies was assessed by consistency of findings for similar designs and outcomes, accounting for quality and risk of bias.

\section{Meta-analysis}

Studies providing baseline or longitudinal data on cytokine concentrations in depressed and non-depressed patients were included in the meta-analysis and bivariate subgroup analysis. A minimum of two studies per inflammatory marker were considered sufficient for meta-analysis to be carried out ${ }^{25}$. For continuous data outcomes a weighted mean difference and $95 \%$ confidence intervals (CIs) were calculated using a random effects model which also allows suspected heterogeneity between studies ${ }^{26}$. Forest plots were generated, and pooled effect sizes were calculated using Comprehensive Meta-Analysis (CMA) version $3{ }^{27}$. The standardised 
mean-difference (SMD) was computed from studies providing means and standard deviations (SD) for cytokine concentrations in depressed and non-depressed patients. Where required SD were calculated from CIs or standard errors (SE) using a verified formula ${ }^{25}$. If a study provided a median, interquartile range (including the first and third quartile) and sample size, this was converted into an estimated sample mean and SD using a formula ${ }^{25}$. Where studies provided a correlation coefficient and a sample $\mathrm{N}$ instead of a means and $\mathrm{SD}$, this was entered into CMA to compute the effect size. If studies did not provide means and SD or correlation coefficient but presented a standardised/unstandardised regression coefficient with SD and sample n's for depressed and non-depressed patients with $95 \%$ CIs, these were entered into CMA to compute the effect size ${ }^{28}$. All effect sizes were calculated such that positive values demonstrate higher levels of inflammatory markers in depressed patients and negative values indicated the opposite. Where adequate data was not provided, study authors were contacted for additional data and were given 3 weeks to respond.

To investigate heterogeneity, subgroup and sensitivity analysis was carried out for all inflammatory markers. Within group heterogeneity was assessed using Higgin's $\mathrm{I}^{2}$ with $\mathrm{a} \geq 50 \%$ cut-off for 'substantial heterogeneity' ${ }^{25}$. Cochrane's $Q$ with $p$ values $<0.05$ was also reported to indicate significant within and between group heterogeneity. Likely sources of heterogeneity investigated included the way in which depression was defined (structured clinical interview or validated self-report depression tool) and the type of data provided by studies which was used to calculate the effect size. Sensitivity analyses was carried out on study design for each inflammatory marker (cross sectional; longitudinal; RCT) and study quality (poor; fair; good). Meta-regression investigating age, gender and ethnicity was considered for inflammatory markers with more than 10 studies, however this data was reported for the total sample rather than for depressed/non-depressed patients rendering it inappropriate ${ }^{25}$. Publication bias using 
Egger's test for funnel plot symmetry was assessed for inflammatory makers containing more than 10 studies (CRP; IL-6; TNF-a). In all analyses, statistical significance was set at $p \leq 0.05$.

\section{Results}

The searches yielded 8,182 hits (see PRISMA, Figure 1). Fifty-three studies met our inclusion criteria, and seven additional studies were identified by citation tracking; 61 entered the review.

\section{Study characteristics}

Studies which are included in the review are outlined in table 1. Two studies used an RCT design, and two reported on the effects of the intervention on inflammatory biomarkers and depression severity in comparison with controls ${ }^{29,30}$. Four studies used an non-randomised intervention design, three were cohort (CS), and 53 were cross sectional (CSS).

The largest number of studies were carried out in East Asia (20/60) and Europe (19/60), with other studies conducted in the USA (4/60) and South America (2/60). The number of participants ranged from 28 to 917. Most studies included haemodialysis (HD) patients (35/60), followed by mixed sample of HD and Peritoneal dialysis (PD) patients (11/60), PD patients (10/60), CKD patients (2/60) and mixed sample of CKD and HD or PD patients $(2 / 60)$

Most studies used self-report depression screening scales to measure the presence of depressive symptoms or severity (52/60); choice of which varied considerably (Beck Depression Inventory (BDI), Patient Health Questionnaire (PHQ)-9, Hamilton Depression Rating Scale (HAM-D) or Hospital Anxiety and Depression Scale (HADS-D), Center for Epidemiological Studies Depression (CESD), Zung Self-rating Depression Scale (SDS), Allgemeine Depression-Skala (ADS-L), Mental Health Inventory (MHI)-5, Geriatric Depression Scale (GDS)) and 8 (8/60) 
studies used a structured clinical interview for depression diagnosis. The majority of studies measured only one biomarker (35/60); CRP was measured most often (54/60), followed by IL$6(28 / 60)$, TNF-a (15/60), IL-10 (7/60) and a range of other biomarkers (IL-1 $\beta$, WBC count, fibrinogen, IL-17, IL-18 and IL-12p70; note hs-CRP and CRP are referred to as CRP).

Thirty studies (30/60) aimed at investigating the association between inflammatory markers and depression in ESRD/CKD patients or aimed at investigating the effects of an intervention on depressive symptoms associated with inflammatory markers. These studies are referred to as 'fit for purpose' in the current review (see Table 1). The remaining thirty (30/60) studies collected and analysed data on depression and inflammatory markers for other purposes.

\section{Cross-sectional associations between depressive symptoms (DS), Major Depression (MD), and inflammatory biomarkers}

Forty-four studies analysed cross-sectional data on major depression MD/DS and CRP levels. Most studies (39/44) used a self-report screening tool to measure DS, and five (5/44) used a structured clinical interview to diagnose MD.

Fifteen studies (15/44) reported significant associations between CRP and MD/DS. Five studies diagnosed patients with MD using a structured clinical interview ${ }^{31,35}$. Of these, three reported no significant differences in CRP levels between depressed and non-depressed HD/PD patients 31,33. Two studies reported significantly higher CRP levels in depressed compared to the nondepressed group ${ }^{34,35}$, with one study including mixed samples of ESRD and CKD patients and did not exclude patients with infections or inflammatory diseases.

Studies which used the BDI threshold score $\geq 14$, tended to report non-significant associations between DS and CRP; the majority were carried out in Europe and West Asia (11/15). Exceptions are studies by Bossola, et al. ${ }^{36}$ and Micozkadioglu, et al. ${ }^{37}$ which reported nonsignificant findings on the BDI and the CDI (truncated cognitive version of the BDI) with a 
threshold score of $\geq 10^{3637}$. These studies with lower cut-off scores reported a greater proportion of patients with depressive symptoms (38/44) and longest periods of dialysis ( $\geq 12$ months) compared to other studies. Studies which used other depression scales such as the HADS-D (score $\geq 8$ ), CES-D (score $\geq 16$ ), GDS (score $>5$ ), PHQ 2 and MHI-5 reported non-significant associations between depression and CRP.

There were 13 studies reporting significant associations between CRP and DS. ${ }^{38-50}$ Eight of these were fit for purpose studies. Three were from East Asia and using a BDI cut of score of $>14$, they found elevated CRP levels in DS compared to the no DS group ${ }^{39,40,47}$. Three others found the same using a BDI cut-off score of $>10$ or $>13^{41,47,48}$. All studies using the HAMD or Zung depression scale reported significantly higher CRP levels amongst those with DS ${ }^{38,40}$ 44,46. Studies which used the ADS-L ( $>23)$ or the PHQ $9(>10)$ also reported significant positive correlations between depression scores and CRP 45,49 .

Conclusion: There is evidence of CRP being related to $D S$ and $M D$, across a range of measures of depression and thresholds. Studies designed to assess this association (fit for purpose studies) were more likely to demonstrate an association.

Associations between IL-6 and depression

Twenty studies present cross-sectional analysis on the association between depression and IL$6^{32,33,36,41,47,49,51-64}$. Of these, the majority were fit for purpose $(17 / 20,85 \%)$. Twelve of these $(60 \%)$ reported significant associations between depression and IL-6. Four studies of HD/PD and CKD patients using self-report tools (MHI-5, HADS-D and GDS) and found nonsignificant associations between DS and IL-6 ${ }^{51-53,55}$. Two studies which used the SCID to diagnose MD reported contradictory results ${ }^{32,33}$. Kalender, et al. ${ }^{32}$ found no significant association whereas Wang, et al. ${ }^{33}$ reported significantly higher IL-6 levels in depressed 
compared to non-depressed patients. Kalender, et al. ${ }^{32}$ used a sample of HD patients (with exclusion of acute illnesses) from Turkey, whilst Wang, et al. ${ }^{33}$ used a sample of PD (without exclusion of acute illnesses) patients from Taiwan.

Out of the 12 studies reporting a significant association between IL-6 and DS 33364749 56-63 , the majority were fit for purpose studies $(9 / 12,75 \%) ; 66 \%$ used the BDI to measure DS. Studies reported a significant result irrespective of the BDI cut off scores (ranging from $\geq 10$ to $\geq 16$ ). Studies which used depression scales such as the ADL-S, HADS-D and the Zung self-rating depression scale also reported significant findings in both HD and PD patients; these studies were comparable in terms of sample composition (mean age; inclusion/exclusion criteria).

Conclusion: In relation to associations between IL-6 levels and DS, the majority reporting significant associations were fit for purpose studies. The evidence of an association between MD and IL-6 is inconclusive as one of the two studies included patients with acute illness which could explain the positive association.

Associations between TNF- $\alpha$ and depression

Twelve studies presented cross sectional data on the association between depression and TNF$\alpha^{32,33,41,43,53-55,62,64-67}$. Eleven studies reported non-significant findings $32,33,41,53-55,62,64-67$. Four studies that used the SCID to diagnose depression report similar proportions of depressed patients $(26 \%-24 \%)$ despite the difference in dialysis modality (HD \& PD). Neither reported any significant findings between MD and TNF- $\alpha$ levels ${ }^{32,33,65,66}$. Most studies which used BDI to define depressive symptoms regardless of cut off scores, reported a non-significant finding. An exception to this is the study by Ko, et al. ${ }^{43}$, which was the only study to find a significant finding between depressive symptoms and TNF- $\alpha$ in PD patients using the BDI $(>15)$.

Conclusion: There is little evidence of an association between MD/DS and TNF- $\alpha$. 
Associations between IL-10 and depression

Seven studies analysed cross sectional data on the association between depression and IL-10 41 , $43,47,53,55,62,64$. Five studies (83\%) reported non-significant results between IL-10 levels and depressive symptoms $47,53,55,62,64$. Most studies used a single centre sample of dialysis patients (HD or PD or mixed sample). All studies used a depression rating scale (BDI or HADS-D) with various cut-off scores and were either of fair or good quality, because of a lack of information on non-responders and sample justification.

Two studies reported significant cross sectional associations between DS and lower IL 10 levels in non-depressed patients ${ }^{41}{ }^{43}$. Both studies used the BDI to measure DS with Ko, et al. ${ }^{43}$ using a higher BDI cut-off point (BDI>15). Both studies are rated as good quality, however the inclusion and exclusion criteria for patients in Haverkamp, et al. ${ }^{41}$ weren't as stringent as Ko, et al. ${ }^{43}$, where patients with inflammatory diseases were included which could affect the levels of inflammatory cytokines.

Conclusions: There is little evidence of an association between IL-10 and depression in ESRD/CKD population. The one positive study included patients with other conditions suggesting this may explain the negative association.

Associations between other inflammatory markers and depression

Guenzani, et al. ${ }^{53}$ was the only study to assess cross-sectional findings associations between IL-17 and IL-12p70 and depressive symptoms in CKD patients. The study reported a nonsignificant association for IL-17 and a significant association for IL-12p70, and depressive symptoms. 
Conclusion: The evidence is inconclusive.

Associations between fibrinogen and depressive symptoms (DS)

Three studies report on cross-sectional associations between DS and fibrinogen 364357 . Three report conflicting findings. Two studies reported significantly higher fibrinogen levels in HD patients with depressive symptoms compared to those with no depressive symptoms; these findings were across a range of cut-off scores on the BDI ( $\geq 10$ or $>14)$ and varying periods of time on dialysis ( $\geq 12$ or $>6$ months $)^{3657}$. Ko, et al. ${ }^{43}$ reported no significant differences in fibrinogen levels in PD patients with or without depressive symptoms using the BDI with a cut off of $>15$.

Conclusions: The evidence is inconclusive.

Associations between White Blood Cell (WBC) count and depressive symptoms

Two studies examined cross-sectional associations between depressive symptoms and WBC count and reported contradictory findings ${ }^{5168}$. Both studies were carried out in the US using large samples recruited from multiple dialysis units, they are similar in study quality and reported similar percentages for patients with depressive symptoms despite using different depression scales. Boulware, et al. ${ }^{51}$ reported significantly higher WBC count in patients with depressive symptoms using the MHI-5 ( $\geq 52)$, whereas Fan, et al. ${ }^{68}$ reported non-significant results using the CES-D $(\geq 17)$.

Conclusions: There is no evidence of an association. 


\section{Longitudinal associations between depressive symptoms and inflammatory cytokines}

Three studies reported on longitudinal association between DS and CRP 64, 69, 70. The studies were similar in terms of dialysis modality (HD patients), depression definition (BDI) and percentage of females (ranging from 39.4\% - 41\%). Barros, et al. ${ }^{69}$ reported significantly higher CRP levels in the DS patients at the 12-month follow up compared to the no DS group. Similarly, Haverkamp, et al. ${ }^{64}$ reported similar findings to Barros et al. at the 6 month followup but not at the 12 month follow-up. Haverkamp, et al. ${ }^{64}$ found that CRP was an independent predictor for an increase in DS, meaning that when compared with patients with stable or decreased BDI at follow up, patients with increased BDI at follow up had higher baseline serum CRP levels.

Two studies used the BDI to assess for depressive symptoms and failed to report longitudinal associations between depressive symptoms and IL-6 levels. ${ }^{64}$. 0 One study assessed the association between DS and TNF- $\alpha$, IL-10 and IL-1 $\beta$ and reported no significant longitudinal associations between DS and TNF- $\alpha$, IL-10 and IL-1 ${ }^{64}$. Bossola, et al. ${ }^{70}$ reported no significant findings in relation to increased fibrinogen levels in patients with an increased in depressive symptoms at follow-up.

Conclusions: Overall no longitudinal studies show significant associations between DS and the following inflammatory cytokines: IL-6, TNF- $\alpha, I L-10$ and IL-1 $\beta$ in ESRD patients. There is limited evidence of longitudinal associations of $D S$ with CRP.

Effects of antidepressant therapy on inflammatory cytokine concentrations

Six studies which investigated the effects of antidepressant treatment on depression and levels of inflammatory cytokines in ESRD patients on dialysis 29, 30, 65, 66, 71, 72 . All six studies administered SSRI's in depressed HD or PD patients (sertraline, fluoxetine or escitalopram). 
Cilan, et al. ${ }^{65} 66$ diagnosed depression using the SCID whereas the remaining four studies used a depression screening tool HAMD or BDI to define patients with DS.

CRP: Zahed, et al. ${ }^{72}$ reported a significant decrease in CRP levels following 12 weeks of treatment with $50 \mathrm{mg}$ of sertraline.

IL-6: Two RCTs reported on the effects of SSRI's on depressive symptoms and IL-6 levels ${ }^{29}$ ${ }^{30}$. Taraz, et al. ${ }^{29}$ reported significant decrease in DS as well as IL-6 levels following 12 weeks of treatment on $50-100 \mathrm{mg}$ of sertraline. Zhao, et al. ${ }^{30}$ reported no significant findings in IL-6 levels following 18 weeks of treatment on $20 \mathrm{mg}$ escitalopram alone, although IL-6 levels decreased significantly in the escitalopram and aerobic exercise group, with the lowest decrease in the exercise group alone. Three non-randomised interventions studies investigated the effects of antidepressant treatment on depression and IL-6 656671 . All three studies administered SSRI's in depressed HD patients (sertraline, $50 \mathrm{mg}$ or fluoxetine $20 \mathrm{mg}$ ) over an 8-week period. Two studies which diagnosed depression using the SCID reported that sertraline had no significant effect on IL-6 levels in the depressed group following treatment ${ }^{65}{ }^{66}$. Paradoxically, Lee, et al. ${ }^{71}$ reported that depressed patients who responded to fluoxetine were found to have significantly higher levels of IL-6 at follow-up compared to lower levels at baseline.

TNF- $\alpha$ : Taraz, et al. ${ }^{29}$ was the only RCT to investigate the effects of sertraline on DS and TNF$\alpha$ concentrations and although sertraline significantly decreased DS at follow up, there were no significant differences in TNF- $\alpha$ at follow up. Three NRIS investigated the effects of antidepressant treatment (sertraline, $50 \mathrm{mg}$ or fluoxetine $20 \mathrm{mg}$ ) on depression and TNF- $\alpha{ }^{65} 66$ 71. Regardless of the way in which depression was defined, all three studies reported that sertraline or fluoxetine had no significant effect on TNF- $\alpha$ in the depressed group following treatment. Overall these four studies demonstrate no association between DS and TNF- $\alpha$ concentrations following SSRI treatment in the ESRD population. 
IL-10, IL-2 and WBC: Taraz, et al. ${ }^{29}$ was the only RCT to report on the effects of sertraline on DS and IL-10 levels and reported no significant findings in HD patients. The same finding was also reported with regards to WBC count. Zahed, et al. ${ }^{72}$ also reported no significant findings between WBC and DS following 12 weeks of sertraline treatment. Lee, et al. ${ }^{71}$ was the only study to report on the effects of SSRI's on DS and IL-2 levels and found no non-significant findings. Due to the small number of studies reporting on IL-10, IL-2 and WBC count, it is not possible to draw conclusions on the effects of SSRI's on DS and these inflammatory biomarkers.

Conclusion: There is some evidence of a reduction of CRP or IL6, and perhaps interaction with other intervention components, but this is not convincing. However, there is no evidence of an effect on $T N F-\alpha, I L-10, I L-2$, and $W B C$.

\section{Effects of dietary supplements on depressive symptoms and inflammatory cytokine concentrations}

One study reports on the effects of non-antidepressant therapy on inflammatory cytokine concentrations Zhang, et al. ${ }^{73}$ a NRIS which investigated the effects of vitamin D supplementation on depressive symptoms and CRP in HD patients. The study reported significant cross-sectional associations between CRP and depressive symptoms (BDI $\geq 16$ ), however, found although vitamin D supplements reduced levels of CRP at follow up there were no consequent reductions in DS.

Conclusion: There is no evidence that supplements impact on inflammatory markers and DS.

\section{Longitudinal meta-analysis}




\section{CRP}

Two longitudinal studies ${ }^{6469}$ using a validated self-report depression tool contained four analyses that were appropriate for meta-analysis. No significant findings were found between CRP concentrations in depressed and non-depressed groups at baseline $\left(\mathrm{SMD}=0.12, \mathrm{CI}^{95}=-\right.$ $0.36-0.60, \mathrm{p}=0.62)$ or at follow-up $\left(\mathrm{SMD}=-0.19, \mathrm{CI}^{95}=-0.85-0.46, \mathrm{p}=0.57\right)$. In subgroup and sensitivity analyses (see Table ???), no significant differences were detected by the timeframe (baseline vs. 12 months follow-up) or quality of the studies to explain heterogeneity.

Conclusion: there is no evidence of CRP being associated with DS and MD.

\section{Cross-sectional meta-analyses}

49 analyses of 45 cross-sectional studies found significantly higher concentrations of CRP in the depressed group compared to non-depressed group $\left(\mathrm{SMD}=0.50, \mathrm{CI}^{95}=0.27-0.72, \mathrm{p}<0.0001\right)$ $31-51,53,54,57,59,62-64,66,68,69,73-86$. Significant heterogeneity was detected $\left(\mathrm{I}^{2}=95 \%\right.$; $\mathrm{Chi}^{2}=1042.29$, $\mathrm{df}=48 ; \mathrm{p}<0.0001 ; \mathrm{Tau}^{2}=0.61$ ); 7 analyses (from 7 studies) found no significant differences in CRP concentrations between depressed and non-depressed group when depression was defined using a structured clinical interview $\left(\mathrm{SMD}=0.56, \mathrm{CI}^{95}=-0.06-1.18, \mathrm{p}=0.08\right)$, with moderate significant heterogeneity $\left(\mathrm{I}^{2}=55 \% ; \mathrm{Chi}^{2}=13.44, \mathrm{df}=6 ; \mathrm{p}=0.037 ; \mathrm{Tau}^{2}=0.07\right){ }^{31-35} 6674.42$ analyses (from 38 studies) found significant differences in CRP concentrations between depressed and non-depressed groups when depression was defined using a validated self-report depression tool $\left(\mathrm{SMD}=0.49, \mathrm{CI}^{95}=0.24-0.73, \mathrm{p}<0.001\right)$, with significant considerable heterogeneity $\left(\mathrm{I}^{2}=96 \%\right.$; $\mathrm{Chi}^{2}=1028.42, \mathrm{df}=41 ; \mathrm{p}<0.0001$; $\left.\mathrm{Tau}^{2}=0.66\right){ }^{36-515354575962-6468697375-}$ ${ }^{86}$. However, the comparison between structured clinical interviews with depression defined using a validated self-report measure reported no significant between-group differences 
$\left(\mathrm{Chi}^{2}=0.47, \mathrm{df}=1 ; \mathrm{p}=0.83\right)$. No significant findings were reported for total between group heterogeneity when comparing effect size calculation formats or study quality (see table $4 b$ ).

There is some evidence in cross-sectional studies of associations of CRP with MD/DS, but this is inconsistent and there is much heterogeneity.

Cross sectional associations between IL-6 and depression

Twenty-nine analyses from 27 studies were appropriate for inclusion in the meta-analyses investigating cross-sectional associations between IL-6 and depression 30, 32, 33, 36, 41, 46, 47, 49, 5154, 56-59, 61-67, 80, 82, 87 . These included structured clinical interviews and validated self-report depression tools and found significantly higher concentrations of IL-6 in the depressed group compared to non-depressed group (pooled standardised mean difference $=0.67, \mathrm{CI}^{95}=0.35$ 0.99, $\mathrm{p}<0.001)$. There was significant heterogeneity $\left(\mathrm{I}^{2}=96 \% ; \mathrm{Chi}^{2}=696.72, \mathrm{df}=28 ; \mathrm{p}<0.001\right.$; $\left.\operatorname{Tau}^{2}=0.71\right)$.

Out of the 29 analyses, 4 found no significant differences in IL-6 concentrations between depressed and non-depressed group when depression was defined using a structured clinical interview (pooled standardised mean difference $=0.23, \mathrm{CI}^{95}=-0.66-1.12, \mathrm{p}=0.62$ ) ${ }^{32,33,65,66}$. All 4 analyses showed no significant heterogeneity $\left(\mathrm{I}^{2}=0 \% ; \mathrm{Chi}^{2}=1.10, \mathrm{df}=3 ; \mathrm{p}=0.78\right.$; $\left.\mathrm{Tau}^{2}=0.00\right)$.

Twenty five analyses (from 22 studies) found significant differences in IL-6 concentrations between depressed and non-depressed groups when depression was defined using a validated self-report depression tool (pooled standardised mean difference $=0.74, \mathrm{CI}^{95}=-0.39-1.08$, $\mathrm{p}<0.001) 29,30,36,41,46,47,49,51-54,56-61,63,64,67,80,82$. All 25 analyses showed significant considerable heterogeneity $\left(\mathrm{I}^{2}=97 \% ; \mathrm{Chi}^{2}=692.92, \mathrm{df}=24 ; \mathrm{p}<0.001 ; \mathrm{Tau}^{2}=0.75\right)$. 
Comparison of total between group heterogeneity comparing structured clinical interviews with depression defined using a validated self-report measure reported no significant heterogeneity $\left(\mathrm{Chi}^{2}=1.08, \mathrm{df}=1 ; \mathrm{p}=0.30\right)$.

Significantly higher IL-6 concentrations in depressed were found when effect sizes were calculated using both means and standard deviations and correlation analysis (see table $5 \mathrm{~b}$ ). However significant heterogeneity was found in analyses using means and SD and correlation analysis. Twenty six analyses were cross sectional and 3 analyses were RCT's ${ }^{30}$. Regardless of study design, both cross sectional and RCT data reported significantly higher IL-6 concentrations in depressed compared to non-depressed groups (see table 5b). Significant considerable heterogeneity was reported for cross-sectional studies but not RCT's, furthermore total between group heterogeneity was found to be significant when comparing cross-sectional to RCT's (see table 4b). Only good quality cross sectional studies (21 analyses) reported significantly higher concentrations of IL-6 in depressed compared to non-depressed groups (see table 4 b) $33,36,41,46,52-54,56-59,62-67,80,82,87$. These results were not replicated in fair quality studies 32, 47, 49, 61; both fair and good quality studies reported considerable heterogeneity (see table 5b).

Conclusion: Meta-analyses of cross-sectional studies show some support of an association between IL6 and MD/DS, irrespective of the measurement method, but there is considerable heterogeneity.

Cross-sectional associations between TNF-a and MD/DS

Eleven analyses from 11 studies were included in the meta-analyses $32,33,41,43,53,54,62,64-67$. These 11 analyses included structured clinical interviews and validated self-report depression tools, and found no significant differences in TNF-a concentrations in the depressed group compared to non-depressed group (pooled standardised mean difference $=0.38, \mathrm{CI}^{95}=-0.02$ - 
$0.78, \mathrm{p}=0.07)$. All 11 analyses showed significant heterogeneity $\left(\mathrm{I}^{2}=93 \%\right.$; $\mathrm{Chi}^{2}=139.03, \mathrm{df}=10$; $\left.\mathrm{p}<0.001 ; \mathrm{Tau}^{2}=0.4\right)$

Four of 11 analyses (from 4 studies) found no significant differences in TNF-a concentrations between depressed and non-depressed group when depression was defined using a structured clinical interview (pooled standardised mean difference $=0.01, \mathrm{CI}^{95}=-0.72-0.73, \mathrm{p}=0.99$ ) ${ }^{32,33}$ 65, 66. All 4 analyses showed no significant differences in heterogeneity $\left(\mathrm{I}^{2}=0 \%\right.$; $\mathrm{Chi}^{2}=2.22$, $\left.\mathrm{df}=3 ; \mathrm{p}=0.53 ; \mathrm{Tau}^{2}=0.00\right)$.

Out of 11 analyses, 7 (from 22 studies) found significant differences in TNF-a concentrations between depressed and non-depressed groups when depression was defined using a validated self-report depression tool (pooled standardised mean difference $=0.52, \mathrm{CI}^{95}=0.07-1.12$, $\mathrm{p}=0.03) 41,43,53,54,62,64,67$. All 7 analyses showed significant heterogeneity $\left(\mathrm{I}^{2}=96 \%\right.$; $\left.\mathrm{Chi}^{2}=136.58, \mathrm{df}=6 ; \mathrm{p}<0.0001 ; \mathrm{Tau}^{2}=0.51\right)$.

Comparison of total between group heterogeneity comparing structured clinical interviews with depression defined using a validated self-report measure reported no significant heterogeneity $\left(\mathrm{Chi}^{2}=1.65, \mathrm{df}=1 ; \mathrm{p}=0.20\right)$.

The type of effect size did not account affect the findings (see table 6b). Both fair and good quality studies reported non-significant findings in TNF-a concentrations between depressed and non-depressed groups with significant heterogeneity reported for good quality studies.

Conclusions: There is some but inconsistent evidence of TNF-a being associated with DS/MD, but significant heterogeneity.

Cross-sectional associations between IL-10 and depression 
Six analyses from 6 studies were appropriate for inclusion in the meta-analyses investigating cross sectional associations between IL-10 and depression $41,43,47,53,62,64$. All used a validated self-report measure to define depression. IL-10 concentrations were significantly lower in the depressed compared to the non-depressed group (pooled standardised mean difference $=-0.57$, $\left.\mathrm{CI}^{95}=-1.09-0.06, \mathrm{p}<0.0001\right)$. All 6 analyses showed significant heterogeneity $\left(\mathrm{I}^{2}=95 \%\right.$; $\left.\mathrm{Chi}^{2}=95.02, \mathrm{df}=5 ; \mathrm{p}<0.0001 ; \mathrm{Tau}^{2}=0.38\right)$. Effect size calculation format showed no influence on the findings (see table $7 b$ ). Significant heterogeneity was only reported for effect size calculations using means and SD. Only good quality studies (5 analyses) reported significantly lower concentrations of IL-10 in depressed compared to non-depressed groups (see table 7b) ${ }^{41}$ $43,53,62,64$.

Conclusion: IL-10 seems to be negatively associated with MD/DS, with considerable heterogeneity.

Cross-sectional associations between IL-1B and depression

Three analyses from 3 studies were appropriate for inclusion (G. L. Haverkamp et al., 2018; G. L. G. Haverkamp et al., 2019; et al., 2012). All used a validated self-report measure to define depression. There were no significant differences in IL-1B concentrations in depressed compared to the non-depressed group (pooled standardised mean difference $=-0.01, \mathrm{CI}^{95}=-0.13-$ $0.11, \mathrm{p}=0.93)$. All 3 analyses showed no significant heterogeneity $\left(\mathrm{I}^{2}=0 \%\right.$; $\mathrm{Chi}^{2}=0.98, \mathrm{df}=2$; $\left.\mathrm{p}=0.61 ; \mathrm{Tau}^{2}=0.00\right)$. All 3 studies were of good quality. Effect size calculation format reported no significant influence on reported IL-1B concentrations in depressed compared to nondepressed groups (see table 9b).

Conclusion: IL-8 shows no associations with MD/DS.

Cross-sectional associations between fibrinogen and depression 
Three analyses from 3 studies were appropriate for inclusion ${ }^{36,43,70}$. All used a validated selfreport measure to define depression. Significantly higher concentrations of fibrinogen were reported in depressed compared to non-depressed group (pooled standardised mean difference= $\left.0.52, \mathrm{CI}^{95}=0.26-0.78, \mathrm{p}<0.0001\right)$. There was no significant heterogeneity $\left(\mathrm{I}^{2}=0 \% ; \mathrm{Chi}^{2}=0.69\right.$, $\mathrm{df}=2 ; \mathrm{p}=0.71 ; \mathrm{Tau}^{2}=0.00$ ). Effect size calculation format showed no influence ( see table $10 \mathrm{~b}$ ). Conclusion: fibrinogen appears to be positively correlated with MD/DS.

Cross sectional associations between WBC count and depression

Two analyses from 2 studies were appropriate for inclusion ${ }^{51,68}$. Both used a validated selfreport measure to define depression. Significantly higher concentrations of WBC count were reported in depressed compared to non-depressed group (pooled standardised mean difference $=$ $\left.0.13, \mathrm{CI}^{95}=0.00-0.26, \mathrm{p}=0.04\right)$. There was no significant heterogeneity $\left(\mathrm{I}^{2}=0 \% ; \mathrm{Chi}^{2}=0.66\right.$, $\mathrm{df}=1 ; \mathrm{p}=0.42 ; \mathrm{Tau}^{2}=0.00$ ). Only one fair quality study reported significantly higher levels of WBC count in depressed compared to non-depressed group (see table 11b). There was one good quality study which reported no significant differences in WBC levels (see table 11b). Furthermore, there were no significant findings reported for total between group heterogeneity for study quality (see table 11b).

Conclusion: the evidence on WBC and depression is inconclusive.

\section{Discussion}

Inflammation may be an important mediator in developing mood disorders and research on antiinflammatories offer the potential for more precise treatment options, with fewer adverse effects or polypharmacy ${ }^{88}$. Furthermore, the presence of inflammation may help predict anti- 
depressant response. ${ }^{89}$ Depressive illness in CKD and ESKD is associated with shorter life expectancy, and when related to inflammation, may make the treatment of depression more complex ${ }^{90}$. However, some people at risk for depression due to family history or living with adversity may be especially vulnerable to future depression and multiple health problems. Therefore, understanding the role of inflammation as a mechanism and possible therapeutic target or predictor, warrants better evidence of the relationship between depression and inflammation in renal disease.

Surprisingly, we found little evidence that inflammatory markers are associated with depression in patients with ESKD. Existing evidence may be related to co-morbidities, as this was present in the findings on CRP in cross-sectional, longitudinal and anti-depressant response studies. IL6 also shows positive associations in cross-sectional and anti-depressant studies but not longitudinal studies. More generally, there is an absence of studies that look at inflammatory markers, and those that do mostly show no associations for TNF-alpha, WBC, IL17, IL12p70, IL16 ,IL2, IL10; fibrinogen, in a pooled analysis, shows significant associations, although one of the two studies was not showing a convincing association with depression.

We identified marked heterogeneity in study designs, but overall the type of outcome measure used (self-report vs structured instrument), duration of dialysis, methods to measure effect size appeared to have some influence. Future studies should focus on CRP and IL6, make more explicit whether there are co-morbid medical conditions, and include measures of a range of severity of depressive illness in ESKD. Longitudinal studies are lacking. More research is needed to investigate inflammatory markers as drivers of depression generally, in the absence of medical illness, and make comparisons with populations with medical conditions, and ESKD specifically. Understanding ESKD and multimorbidity, and the health consequences require more research. Indeed, ESKD commonly arises in patients experiencing hypertension and diabetes; and depressive illness is known to be a co-morbid complication of medical disorders. 
Studies might compare inflammatory markers insamples with depression + multimorbidity including ESKD; depression + ESKD only; and people with neither.

The timing of onset of depressive illness is also important to clarify, as a previous history of depression may predispose to later depression in the face of a medical condition or life event with little specificity for ESKD and related inflammation. Indeed, histories and contemporary experiences of adversity and trauma also make depression more likely and raise inflammatory

markers. Furthermore, ESKD and dialysis are demanding and may lead to adjustment reactions, and pessimism that may not meet criteria for depression but may reflect other life stressors, such as social isolation, fear of loss and death, concerns about dependents, and uncertainty, not to mention the need for lifetime dialysis and a schedule which is dictated by health needs. This will make enjoyment of everyday life more difficult, for example, taking holidays or travel and risks of new onset conditions complicated and already challenging medical condition. Psychological flexibility and adjustment to the diagnosis and treatment are predictors of better outcomes, and might be the mechanism by which therapeutic efforts might be helpful ${ }^{92}$. Indeed, acceptance and commitment therapy encourages psychological flexibility and is being tested in many chronic conditions including cancer survivors, and may have a place in the care of dialysis patients living with uncertainty ${ }^{93}$.

\section{Limitations}

There is limited evidence and few studies that reported in similar samples and on the same inflammatory markers. Longitudinal and experimental studies are needed, testing psychological interventions, social support, and anti-inflammatories. Many studies were not fit for purpose. Most studies were not designed to evaluate our research questions. Larger multicentre studies 
are needed focusing on specific patient groups, and those with specific co-morbidities. limitations of the review and the current literature was needed 


\section{Conflict of Interests: None}

Author contributions: Simone Jayakumar (SJ), a PhD candidate, with Stacey Jennings (JS), undertook searches, screening and extraction under supervision by Bhui (PI), Carvalho (CI) and Hosang. Jayakumar conducted the meta-analysis and produced an early draft. Kristoffer Halvorsrud gave methodological advice and critically assessed the meta-analysis. All authors contributed to the commenting on the MS, interpretation of the results and revisions of the MS. Livia Carvalho and Kamaldeep Bhui were responsible for the conception and design and funding application of the original larger programme, and the review, interpretation, supervision and writing of the MS. All authors have approved the final version.

Acknowledgements: We would like to thank Jessica Schofield for her assistance in full text and abstract screening. Thanks to Fiona Loud and Brian Gracey who commented on the review protocol and were part of the original funding application for the larger programme of work. We would also like to thank the authors who provided full text access and further data to the papers included in the review. We thank colleagues who provided comments on the MS: Roisin Mooney, Georgina Hosang, Magdi Yaqoob. 


\section{References}

1. Levey AS, Eckardt KU, Dorman NM, et al. Nomenclature for kidney function and disease: report of a Kidney Disease: Improving Global Outcomes (KDIGO) Consensus Conference. Kidney Int 2020;97(6):1117-29. doi: 10.1016/j.kint.2020.02.010 [published Online First: 2020/05/16]

2. Harris DCH, Davies SJ, Finkelstein FO, et al. Increasing access to integrated ESKD care as part of universal health coverage. Kidney Int 2019;95(4s):S1-s33. doi: 10.1016/j.kint.2018.12.005 [published Online First: 2019/03/25]

3. Global, regional, and national burden of chronic kidney disease, 1990-2017: a systematic analysis for the Global Burden of Disease Study 2017. Lancet 2020;395(10225):70933. doi: 10.1016/s0140-6736(20)30045-3 [published Online First: 2020/02/18]

4. Foreman KJ, Marquez N, Dolgert A, et al. Forecasting life expectancy, years of life lost, and all-cause and cause-specific mortality for 250 causes of death: reference and alternative scenarios for 2016-40 for 195 countries and territories. Lancet 2018;392(10159):205290. doi: 10.1016/s0140-6736(18)31694-5 [published Online First: 2018/10/21]

5. Liyanage $\mathrm{T}$, Ninomiya $\mathrm{T}$, Jha $\mathrm{V}$, et al. Worldwide access to treatment for end-stage kidney disease: a systematic review. Lancet 2015;385(9981):1975-82. doi: 10.1016/s01406736(14)61601-9 [published Online First: 2015/03/18]

6. Ekrikpo UE, Kengne AP, Bello AK, et al. Chronic kidney disease in the global adult HIVinfected population: A systematic review and meta-analysis. PLoS One 2018;13(4):e0195443. doi: 10.1371/journal.pone.0195443 [published Online First: 2018/04/17]

7. Lunyera J, Mohottige D, Von Isenburg M, et al. CKD of Uncertain Etiology: A Systematic Review. Clin J Am Soc Nephrol 2016;11(3):379-85. doi: 10.2215/cjn.07500715 [published Online First: 2015/12/30]

8. Palmer S, Vecchio M, Craig JC, et al. Prevalence of depression in chronic kidney disease: systematic review and meta-analysis of observational studies. Kidney Int 2013;84(1):179-91. doi: 10.1038/ki.2013.77 [published Online First: 2013/03/15]

9. Palmer SC, Vecchio M, Craig JC, et al. Association between depression and death in people with CKD: a meta-analysis of cohort studies. Am J Kidney Dis 2013;62(3):493-505. doi: 10.1053/j.ajkd.2013.02.369 [published Online First: 2013/04/30]

10. Farrokhi F, Abedi N, Beyene J, et al. Association between depression and mortality in patients receiving long-term dialysis: a systematic review and meta-analysis. Am J Kidney Dis 2014;63(4):623-35. doi: 10.1053/j.ajkd.2013.08.024 [published Online First: 2013/11/05]

11. Kimmel PL. Psychosocial factors in dialysis patients. Kidney Int 2001;59(4):1599-613. doi: 10.1046/j.1523-1755.2001.0590041599.x [published Online First: 2001/03/22]

12. Gupta J, Mitra N, Kanetsky PA, et al. Association between albuminuria, kidney function, and inflammatory biomarker profile in CKD in CRIC. Clin J Am Soc Nephrol 2012;7(12):1938-46. doi: 10.2215/cjn.03500412 [published Online First: 2012/10/02]

13. Gregg LP, Carmody T, Le D, et al. A Systematic Review and Meta-Analysis of Depression and Protein-Energy Wasting in Kidney Disease. Kidney Int Rep 2020;5(3):318-30. doi: 10.1016/j.ekir.2019.12.009 [published Online First: 2020/03/11]

14. Tonelli M, Wiebe N, Guthrie B, et al. Comorbidity as a driver of adverse outcomes in people with chronic kidney disease. Kidney Int 2015;88(4):859-66. doi: 10.1038/ki.2015.228 [published Online First: 2015/07/30]

15. Moher D, Shamseer L, Clarke M, et al. Preferred reporting items for systematic review and meta-analysis protocols (PRISMA-P) 2015 statement. Systematic Reviews 2015;4(1):1. doi: 10.1186/2046-4053-4-1 
16. Simone Jayakumar KB, Jessica Schofield, Amy Ronaldson, Magdi Yaqoob, Livia Carvalho. The association between depression and inflammation in patients with chronic kidney disease(CKD) or End Stage Renal Disease (ESRD): a systematic review of the literature. $2019 \quad$ [Available from: https://www.crd.york.ac.uk/prospero/display record.php?ID=CRD42019141305.

17. Morgan RL, Whaley P, Thayer KA, et al. Identifying the PECO: A framework for formulating good questions to explore the association of environmental and other exposures with health outcomes. Environ Int 2018;121(Pt 1):1027-31. doi: 10.1016/j.envint.2018.07.015 [published Online First: 2018/09/01]

18. Bramer WM, Rethlefsen ML, Kleijnen J, et al. Optimal database combinations for literature searches in systematic reviews: a prospective exploratory study. Systematic Reviews 2017;6(1):245. doi: 10.1186/s13643-017-0644-y

19. Ouzzani M, Hammady H, Fedorowicz Z, et al. Rayyan - a web and mobile app for systematic reviews. Systematic Reviews 2016;5(1):210. doi: 10.1186/s13643-016-03844

20. Sterne JAC, Savović J, Page MJ, et al. RoB 2: a revised tool for assessing risk of bias in randomised trials. Bmj 2019;366:14898. doi: 10.1136/bmj.14898 [published Online First: 2019/08/30]

21. Sterne JA, Hernán MA, Reeves BC, et al. ROBINS-I: a tool for assessing risk of bias in non-randomised studies of interventions. $B M J$ 2016;355:i4919. doi: 10.1136/bmj.i4919

22. The Newcastle-Ottawa Scale (NOS) for Assessing the Quality of Nonrandomised Studies in Meta-Analyses; 2014.

23. Downes MJ, Brennan ML, Williams HC, et al. Development of a critical appraisal tool to assess the quality of cross-sectional studies (AXIS). BMJ Open 2016;6(12):e011458. doi: 10.1136/bmjopen-2016-011458

24. Popay J, Roberts H, Sowden A, et al. Guidance on the conduct of narrative synthesis in systematic reviews. A product from the ESRC methods programme Version 2006;1:b92.

25. Wan X, Wang W, Liu J, et al. Estimating the sample mean and standard deviation from the sample size, median, range and/or interquartile range. BMC Medical Research Methodology 2014;14(1):135. doi: 10.1186/1471-2288-14-135

26. Higgins JP, Thomas J, Chandler J, et al. Cochrane handbook for systematic reviews of interventions: John Wiley \& Sons 2019.

27. Borenstein M, Hedges L, Higgins J, et al. Comprehensive Meta-analysis. Englewood, NJ: Biostat; 2009a. Computer program, version;2

28. Wilson DB. Practical meta-analysis effect size calculator 2001 [Available from: https://campbellcollaboration.org/escalc/html/EffectSizeCalculator-Home.php. accessed 30/06 2020.

29. Taraz M, Khatami MR, Dashti-Khavidaki S, et al. Sertraline decreases serum level of interleukin-6 (IL-6) in hemodialysis patients with depression: results of a randomized double-blind, placebo-controlled clinical trial. Int Immunopharmacol 2013;17(3):91723. doi: 10.1016/j.intimp.2013.09.020 [published Online First: 2013/10/15]

30. Zhao C, Ma H, Yang L, et al. Long-term bicycle riding ameliorates the depression of the patients undergoing hemodialysis by affecting the levels of interleukin-6 and interleukin-18. Neuropsychiatr Dis Treat 2017;13:91-100. doi: 10.2147/ndt.S124630 [published Online First: 2017/01/18]

31. Armaly Z, Farah J, Jabbour A, et al. Major depressive disorders in chronic hemodialysis patients in Nazareth: identification and assessment. Neuropsychiatr Dis Treat 2012;8:329-38. doi: 10.2147/ndt.S31903 [published Online First: 2012/08/14] 
32. Kalender B, Dervisoglu E, Sengul E, et al. Depression, nutritional status, and serum cytokines in peritoneal dialysis patients: is there a relationship? Perit Dial Int 2007;27(5):593-5. [published Online First: 2007/08/21]

33. Wang Y, Liu Y, Lian Y, et al. Efficacy of High-Dose Supplementation With Oral Vitamin D3 on Depressive Symptoms in Dialysis Patients With Vitamin D3 Insufficiency: A Prospective, Randomized, Double-Blind Study. J Clin Psychopharmacol 2016;36(3):229-35. doi: 10.1097/jcp.0000000000000486 [published Online First: 2016/03/30]

34. Choi MJ, Seo JW, Yoon JW, et al. The malnutrition-inflammation-depressionarteriosclerosis complex is associated with an increased risk of cardiovascular disease and all-cause death in chronic hemodialysis patients. Nephron - Clinical Practice 2013;122(1):44-52.

35. Kalender B, Ozdemir AC, Koroglu G. Association of depression with markers of nutrition and inflammation in chronic kidney disease and end-stage renal disease. Nephron Clin Pract 2006;102(3-4):c115-21. doi: 10.1159/000089669 [published Online First: 2005/11/12]

36. Bossola M, Di Stasio E, Giungi S, et al. Fatigue is associated with serum interleukin-6 levels and symptoms of depression in patients on chronic hemodialysis. J Pain Symptom Manage 2015;49(3):578-85. doi: 10.1016/j.jpainsymman.2014.07.009 [published Online First: 2014/08/20]

37. Micozkadioglu H, Micozkadioglu I, Zumrutdal A, et al. Relationship between depressive affect and malnutrition-inflammation complex syndrome in haemodialysis patients. Nephrology (Carlton) 2006;11(6):502-5. doi: 10.1111/j.1440-1797.2006.00664.x [published Online First: 2007/01/04]

38. Bornivelli C, Aperis G, Giannikouris I, et al. RELATIONSHIP BETWEEN DEPRESSION, CLINICAL AND BIOCHEMICAL PARAMETERS IN PATIENTS UNDERGOING HAEMODIALYSIS. Journal of Renal Care 2012;38(2):93-97. doi: https://doi.org/10.1111/j.1755-6686.2012.00259.x

39. Dogan E, Erkoc R, Eryonucu B, et al. Relation between depression, some laboratory parameters, and quality of life in hemodialysis patients. Ren Fail 2005;27(6):695-9. doi: 10.1080/08860220500242728 [published Online First: 2005/12/15]

40. Dong J, Pi H-c, Xiong Z-y, et al. Depression and cognitive impairment in peritoneal dialysis: a multicenter cross-sectional study. American Journal of Kidney Diseases 2016;67(1):111-18.

41. Haverkamp GL, Loosman WL, Schouten RW, et al. Differences in the association of inflammation and tryptophan with depressive symptoms between white and non-white chronic dialysis patients. Gen Hosp Psychiatry 2018;50:76-82. doi: 10.1016/j.genhosppsych.2017.10.003 [published Online First: 2017/10/25]

42. Kim JK, Kim SG, Kim HJ, et al. Serum S100B protein is associated with depressive symptoms in patients with end-stage renal disease. Clin Biochem 2012;45(18):1573-7. doi: 10.1016/j.clinbiochem.2012.08.014 [published Online First: 2012/09/01]

43. Ko GJ, Kim MG, Yu YM, et al. Association between depression symptoms with inflammation and cardiovascular risk factors in patients undergoing peritoneal dialysis. Nephron Clin Pract 2010;116(1):c29-35. doi: 10.1159/000314548 [published Online First: 2010/05/13]

44. Li ZJ, An X, Mao HP, et al. Association between depression and malnutrition-inflammation complex syndrome in patients with continuous ambulatory peritoneal dialysis. Int Urol Nephrol 2011;43(3):875-82. doi: 10.1007/s11255-011-9917-x [published Online First: 2011/03/02] 
45. Malhotra R, Persic V, Zhang W, et al. Tryptophan and Kynurenine Levels and Its Association With Sleep, Nonphysical Fatigue, and Depression in Chronic Hemodialysis Patients. J Ren Nutr 2017;27(4):260-66. doi: 10.1053/j.jrn.2017.01.024 [published Online First: 2017/04/04]

46. Nie XD, Wang Q, Zhang YH, et al. Depression at Baseline Is an Independent Risk Factor for Cognitive Decline in Patients on Peritoneal Dialysis: A Multicenter Prospective Cohort Study. Perit Dial Int 2019;39(5):465-71. doi: 10.3747/pdi.2018.00239 [published Online First: 2019/09/11]

47. Simic Ogrizovic S, Jovanovic D, Dopsaj V, et al. Could depression be a new branch of MIA syndrome? Clin Nephrol 2009;71(2):164-72. doi: 10.5414/cnp71164

48. Gok Oguz E, Akoglu H, Ulusal Okyay G, et al. Serum apelin is associated with affective disorders in peritoneal dialysis patients. Ren Fail 2016;38(7):1059-66. doi: 10.1080/0886022x.2016.1193873 [published Online First: 2016/06/09]

49. Schricker S, Heider T, Schanz M, et al. Strong Associations Between Inflammation, Pruritus and Mental Health in Dialysis Patients. Acta Derm Venereol 2019;99(6):524-29. doi: 10.2340/00015555-3128 [published Online First: 2019/01/24]

50. Su SF, Ng HY, Huang TL, et al. Survey of depression by Beck Depression Inventory in uremic patients undergoing hemodialysis and hemodiafiltration. Ther Apher Dial 2012;16(6):573-9. doi: 10.1111/j.1744-9987.2012.01094.x [published Online First: 2012/11/30]

51. Boulware LE, Liu Y, Fink NE, et al. Temporal relation among depression symptoms, cardiovascular disease events, and mortality in end-stage renal disease: contribution of reverse causality. Clin $J$ Am Soc Nephrol 2006;1(3):496-504. doi: 10.2215/cjn.00030505 [published Online First: 2007/08/21]

52. Brys ADH, Di Stasio E, Lenaert B, et al. Serum interleukin-6 and endotoxin levels and their relationship with fatigue and depressive symptoms in patients on chronic haemodialysis. Cytokine 2020;125:154823. doi: https://doi.org/10.1016/j.cyto.2019.154823

53. Guenzani D, Buoli M, Caldiroli L, et al. Malnutrition and inflammation are associated with severity of depressive and cognitive symptoms of old patients affected by chronic kidney disease. $J$ Psychosom Res 2019;124:109783. doi: 10.1016/j.jpsychores.2019.109783 [published Online First: 2019/08/25]

54. Jong I-C, Tsai H-B, Lin C-H, et al. Close correlation between the ankle-brachial index and symptoms of depression in hemodialysis patients. Int Urol Nephrol 2017;49(8):146370. doi: 10.1007/s11255-017-1598-7

55. Montinaro V, Iaffaldano GP, Granata S, et al. Emotional symptoms, quality of life and cytokine profile in hemodialysis patients. Clin Nephrol 2010;73(1):36-43. doi: 10.5414/cnp73036 [published Online First: 2009/12/31]

56. Alshogran OY, Khalil AA, Oweis AO, et al. Association of brain-derived neurotrophic factor and interleukin-6 serum levels with depressive and anxiety symptoms in hemodialysis patients. General hospital psychiatry 2018;53:25-31. doi: 10.1016/j.genhosppsych.2018.04.003

57. Bossola M, Ciciarelli C, Di Stasio E, et al. Correlates of symptoms of depression and anxiety in chronic hemodialysis patients. Gen Hosp Psychiatry 2010;32(2):125-31. doi: 10.1016/j.genhosppsych.2009.10.009 [published Online First: 2010/03/23]

58. Damayanti I, Nasution HH, Lubis WH. Correlation of Depression Symptoms and Interleukin-6 in Chronic Renal Disease with Regular Hemodialysis Patients. Sumatera Medical Journal 2018;1(1):19-26. doi: 10.32734/sumej.v1i1.442

59. Hung KC, Wu CC, Chen HS, et al. Serum IL-6, albumin and co-morbidities are closely correlated with symptoms of depression in patients on maintenance haemodialysis. 
Nephrol Dial Transplant 2011;26(2):658-64. doi: 10.1093/ndt/gfq411 [published Online First: 2010/07/16]

60. Knuth B, Radtke V, Rocha P, et al. Prevalence of depression symptoms and serum levels of interleukin-6 in hemodialysis patients. Psychiatry and Clinical Neurosciences 2014;68(4):275-82.

61. Sonikian M, Metaxaki P, Papavasileiou D, et al. Effects of interleukin-6 on depression risk in dialysis patients. Am J Nephrol 2010;31(4):303-8. doi: 10.1159/000285110 [published Online First: 2010/02/18]

62. Taraz M, Khatami MR, Gharekhani A, et al. Relationship between a pro- and antiinflammatory cytokine imbalance and depression in haemodialysis patients. Eur Cytokine Netw 2012;23(4):179-86. doi: 10.1684/ecn.2013.0326 [published Online First: 2013/01/31]

63. Uglešić B, Ljutić D, Lasić D, et al. Depression and serum interleukin-6 levels in patients on dialysis. Psychiatr Danub 2015;27(2):168-73. [published Online First: 2015/06/10]

64. Haverkamp GLG, Loosman WL, Schouten RW, et al. Longitudinal Associations Between Inflammation and Depressive Symptoms in Chronic Dialysis Patients. Psychosom Med 2019;81(1):74-80. doi: 10.1097/psy.0000000000000649 [published Online First: 2018/10/30]

65. Cilan H, Oguzhan N, Unal A, et al. Relationship between depression and proinflammatory cytokine levels in hemodialysis patients. Ren Fail 2012;34(3):275-8. doi: 10.3109/0886022x.2011.647292 [published Online First: 2012/01/21]

66. Cilan H, Sipahioglu MH, Oguzhan N, et al. Association between depression, nutritional status, and inflammatory markers in peritoneal dialysis patients. Renal failure 2013;35(1):17-22.

67. Dervisoglu E, Kir HM, Kalender B, et al. Depressive symptoms and proinflammatory cytokine levels in chronic renal failure patients. Nephron Clin Pract 2008;108(4):c2727. doi: 10.1159/000126907 [published Online First: 2008/04/18]

68. Fan L, Sarnak MJ, Tighiouart H, et al. Depression and All-Cause Mortality in Hemodialysis Patients. American Journal of Nephrology 2014;40(1):12-18. doi: 10.1159/000363539

69. Barros A, Costa BE, Mottin CC, et al. Depression, quality of life, and body composition in patients with end-stage renal disease: a cohort study. Braz J Psychiatry 2016;38(4):30106. doi: 10.1590/1516-4446-2015-1681 [published Online First: 2016/02/13]

70. Bossola M, Ciciarelli C, Di Stasio E, et al. Symptoms of depression and anxiety over time in chronic hemodialysis patients. $J$ Nephrol 2012;25(5):689-98. doi: 10.5301/jn.5000042 [published Online First: 2011/10/20]

71. Lee SK, Lee HS, Lee TB, et al. The effects of antidepressant treatment on serum cytokines and nutritional status in hemodialysis patients. J Korean Med Sci 2004;19(3):384-9. doi: 10.3346/jkms.2004.19.3.384 [published Online First: 2004/06/18]

72. Zahed NS, Sharifi M, Karimi M, et al. Impact of sertraline on serum concentration of CRP in hemodialysis patients with depression. J Renal Inj Prev 2016;6(1):65-69. doi: 10.15171 jrip.2017.12

73. Zhang J, Zhang P, Ni X, et al. Vitamin D status in chronic dialysis patients with depression: a prospective study. BMC Psychiatry 2014;14(1):125. doi: 10.1186/1471-244X-14-125

74. Atalay H, Solak Y, Biyik M, et al. Sertraline treatment is associated with an improvement in depression and health-related quality of life in chronic peritoneal dialysis patients. Int Urol Nephrol 2010;42(2):527-36. doi: 10.1007/s11255-009-9686-y

75. Chilcot J, Friedli K, Guirguis A, et al. C reactive protein and depressive symptoms in hemodialysis patients: A questionable association. Hemodialysis International 2017;21(4):542-48. doi: https://doi.org/10.1111/hdi.12500 
76. Güney İ, Biyik M, Yeksan M, et al. Sleep Quality and Depression in Peritoneal Dialysis Patients. Renal Failure 2008;30(10):1017-22. doi: 10.1080/08860220802406419

77. Gyamlani G, Basu A, Geraci S, et al. Depression, screening and quality of life in chronic kidney disease. Am $J$ Med Sci 2011;342(3):186-91. doi: 10.1097/MAJ.0b013e3182113d9e [published Online First: 2011/06/02]

78. Hsu HJ, Chen CK, Hsiao CC, et al. Lack of association between plasma dehydroepiandrosterone sulfate (DHEA-S) levels and depression in hemodialysis patients: a cross-sectional study. Exp Gerontol 2009;44(11):733-9. doi: 10.1016/j.exger.2009.08.009 [published Online First: 2009/09/08]

79. Hsu HJ, Chen CK, Wu MS. Lower prevalence of depression in hemodialysis patients who use polysulfone dialyzers. Am J Nephrol 2009;29(6):592-7. doi: 10.1159/000193144 [published Online First: 2009/01/16]

80. Kusztal M, Trafidło E, Madziarska K, et al. Depressive symptoms but not chronic pain have an impact on the survival of patients undergoing maintenance hemodialysis. Arch Med Sci 2018;14(2):265-75. doi: 10.5114/aoms.2016.59765 [published Online First: 2018/03/30]

81. Mok MMY, Liu CKM, Lam MF, et al. A Longitudinal Study on the Prevalence and Risk Factors for Depression and Anxiety, Quality of Life, and Clinical Outcomes in Incident Peritoneal Dialysis Patients. Perit Dial Int 2019;39(1):74-82. doi: 10.3747/pdi.2017.00168 [published Online First: 2018/07/12]

82. Nowak Ł, Adamczak M, Więcek A. Is inflammation a new risk factor of depression in haemodialysis patients? Int Urol Nephrol 2013;45(4):1121-8. doi: 10.1007/s11255012-0269-y [published Online First: 2012/09/14]

83. Park HC, Yoon HB, Son MJ, et al. Depression and health-related quality of life in maintenance hemodialysis patients. Clin Nephrol 2010;73(5):374-80. doi: $10.5414 / \mathrm{cnp} 73374$

84. Tufan F, Y1ldiz A, Dogan I, et al. Urea to creatinine ratio: a forgotten marker of poor nutritional state in patients undergoing hemodialysis treatment. Aging Male 2015;18(1):49-53. doi: 10.3109/13685538.2014.908281 [published Online First: 2014/04/08]

85. Yavuz R, Yavuz D, Altunoglu A, et al. Depression, Inflammation, and Social Support in Hemodialysis Patients. 2015

86. Park HC, Lee H, Lee JP, et al. Lower residual renal function is a risk factor for depression and impaired health-related quality of life in Korean peritoneal dialysis patients. $J$ Korean Med Sci 2012;27(1):64-71. doi: 10.3346/jkms.2012.27.1.64 [published Online First: 2012/01/06]

87. Knuth B, Radtke V, Rocha P, et al. Prevalence of depression symptoms and serum levels of interleukin-6 in hemodialysis patients. Psychiatry Clin Neurosci 2014;68(4):275-82. doi: 10.1111/pcn.12130 [published Online First: 2014/01/01]

88. Stassen $\mathrm{HH}$, Bachmann $\mathrm{S}$, Bridler $\mathrm{R}$, et al. Inflammatory processes linked to major depression and schizophrenic disorders and the effects of polypharmacy in psychiatry: evidence from a longitudinal study of 279 patients under therapy. Eur Arch Psychiatry Clin Neurosci 2020 doi: 10.1007/s00406-020-01169-0 [published Online First: 2020/07/23]

89. Gregg LP, Carmody T, Le D, et al. Inflammation and Response to Sertraline Treatment in Patients With CKD and Major Depression. Am J Kidney Dis 2020;75(3):457-60. doi: 10.1053/j.ajkd.2019.09.007 [published Online First: 2019/12/24]

90. Schell JO, Bova-Collis R, Eneanya ND. An interdisciplinary approach to dialysis decisionmaking in the CKD patient with depression. Adv Chronic Kidney Dis 2014;21(4):38591. doi: 10.1053/j.ackd.2014.03.012 [published Online First: 2014/06/28] 
91. Bezerra CIL, Silva BC, Elias RM. Decision-making process in the pre-dialysis CKD patients: do anxiety, stress and depression matter? BMC Nephrol 2018;19(1):98. doi: 10.1186/s12882-018-0896-3 [published Online First: 2018/04/29]

92. Iida H, Fujimoto S, Wakita T, et al. Psychological Flexibility and Depression in Advanced CKD and Dialysis. Kidney Med 2020;2(6):684-91 e1. doi: 10.1016/j.xkme.2020.07.004 [published Online First: 2020/12/16]

93. Fernandez-Rodriguez C, Gonzalez-Fernandez S, Coto-Lesmes R, et al. Behavioral Activation and Acceptance and Commitment Therapy in the Treatment of Anxiety and Depression in Cancer Survivors: A Randomized Clinical Trial. Behav Modif 2020:145445520916441. doi: 10.1177/0145445520916441 [published Online First: 2020/04/23] 